\title{
Application of Jacobi Algorithm in Frequency Selective Channels
}

\author{
Pei Xiao, Mathini Sellathurai \\ The Institute of Electronics, Communications and Information Technology \\ Queen's University Belfast, BT3 9DT, United Kingdom \\ E-mail: \{pei.xiao,m.sellathurai\}@ecit.qub.ac.uk
}

\begin{abstract}
In this paper, we apply the Jacobi iterative algorithm to combat intersymbol interference caused by frequency selective channels. An analytical bound of the proposed equalizer is analyzed in order to gain an insight into its asymptotic performance. Due to the error propagation problem, the potential of this algorithm is not reached in an uncoded system. However, its extension to a coded system with the application of the turbo processing principle results in a new turbo equalization algorithm which demonstrates comparable performance with reduced complexity compared to some existing filter based turbo equalization schemes.
\end{abstract}

\section{INTRODUCTION}

In a cellular mobile communications environment, multipath propagation causes dispersion of transmitted signals. The time delay spread causes intersymbol interference (ISI) and degrades system performance. Therefore, equalization methods which can mitigate the effects of ISI must be employed. In [1], [2], etc., the MAP-equalizer [3] was replaced by a linear filter, whose coefficients are adjusted to minimize the mean-square error. It was shown that the performance of this approach is similar to that of the MAP-based receiver, while providing a significant reduction in the computational complexity. In this paper, we first design an equalizer based on the Jacobi algorithm, which has previously been used for multiuser detection in DS-CDMA systems, e.g., in [4]. The algorithm is then extended to a coded system, leading to a new approach to turbo equalization, which further reduces receiver complexity without incurring a performance penalty in most cases compared to the existing filter based algorithms.

A multipath channel can be modeled by an equivalent baseband system where the transmit filter, the channel and the receive filter, are represented by a discrete-time $L$-tap transversal filter with finite-length impulse response $h_{n}=\sum_{l=0}^{L-1} h_{l} \delta_{n-l}$ where $h_{l}$ denotes the complex channel coefficients, which are normalized such that $\sum_{l=0}^{L-1}\left|h_{l}\right|^{2}=1$. The received signal can be formed as

$$
r_{n}=\sum_{l=0}^{L-1} s_{n-l} h_{l}+v_{n},
$$

where $s_{n}=x_{n}+j y_{n}$ denotes the transmitted PSK/QAM symbol (we use QPSK symbol for the purpose of this study) at time instant $n$, and $v_{n}$ is the complex additive white Gaussian noise (AWGN) with zero mean and variance $N_{0}$. The task of the receiver is to detect the transmitted symbols $\left\{s_{n}\right\}$ given the received observation $\left\{r_{n}\right\}$. From (1), we see that the desired symbol is corrupted with ISI and AWGN. An equalizer is needed to combat ISI. Several equalization algorithms have been introduced in the literature, e.g., the minimum mean square error (MMSE) linear equalizer, and the decision feedback equalizer (DFE). Various adaptive algorithms have been proposed for equalizer training, e.g., the least mean square (LMS), and the recursive least square (RLS) [5], square root Kalman (SRK) [6], etc.. Here, we introduce a new equalization algorithm and derive its theoretical performance bound. Our analysis reveals its good potential for removing the detrimental effect of ISI. This approach is later extended to coded systems which leads to a new turbo equalization scheme.

\section{EQUALIZATION BASED ON JACOBi Algorithm}

\section{A. Algorithm derivation}

To simplify the algorithm derivation, we use a 3-tap SUI3 channel specified in IEEE 802.16 standard [7], [8] as an example. It has a tap spacing of 500ns, and maximum tap delay of $1000 \mathrm{~ns}$. The algorithm is then extended to address generic ISI channels. Under the assumption that the transmitted data rate is $4 \mathrm{Mbps}$, the multipath fading can be modeled as a tapped-delay line with adjacent taps spaced equally at the symbol rate. The received signal is formed as $r_{n}=$ $h_{0} s_{n}+h_{1} s_{n-1}+h_{2} s_{n-2}+v_{n}$, where the channel coefficients $h_{0}, h_{1}, h_{2}$ are complex Gaussian random variables and are assumed to remain constant during the transmission of one block of data. The received signal can be written in vector form as

$$
\underbrace{\left[\begin{array}{c}
r_{n} \\
r_{n+1} \\
r_{n+2}
\end{array}\right]}_{\mathbf{r}_{n}}=\underbrace{\left[\begin{array}{ccccc}
h_{2} & h_{1} & h_{0} & 0 & 0 \\
0 & h_{2} & h_{1} & h_{0} & 0 \\
0 & 0 & h_{2} & h_{1} & h_{0}
\end{array}\right]}_{\mathbf{H}} \underbrace{\left[\begin{array}{c}
s_{n-2} \\
s_{n-1} \\
s_{n} \\
s_{n+1} \\
s_{n+2}
\end{array}\right]}_{\mathbf{s}_{n}}+\underbrace{\left[\begin{array}{c}
v_{n} \\
v_{n+1} \\
v_{n+2}
\end{array}\right]}_{\mathbf{v}_{n}} .
$$

The maximum likelihood (ML) estimate of the symbol vector $\mathbf{s}_{n}$ is [9]

$$
\begin{aligned}
\hat{\mathbf{s}}_{n} & =\left(\mathbf{H}^{*} \mathbf{H}\right)^{-1} \mathbf{H}^{*} \mathbf{r}_{n}=\left(\mathbf{H}^{*} \mathbf{H}\right)^{-1} \mathbf{H}^{*}\left(\mathbf{H} \mathbf{s}_{n}+\mathbf{v}_{n}\right) \\
& =\mathbf{s}_{n}+\left(\mathbf{H}^{*} \mathbf{H}\right)^{-1} \mathbf{H}^{*} \mathbf{v}_{n},
\end{aligned}
$$

which is an unbiased estimate of $\mathbf{s}_{n}$ since $\mathrm{E}\left[\hat{\mathbf{s}}_{n}\right]=\mathbf{s}_{n}$. This procedure is computationally complex due to the matrix inverse operation for each symbol vector. The superscript operator ( )* is the conjugate transpose operation when applied to matrices, and simply the conjugate when applied to scalars. To simplify the computation, let us reform (3) as

$$
\begin{aligned}
\hat{\mathbf{s}}_{n} & =\mathbf{s}_{n}+\left(\mathbf{H}^{*} \mathbf{H}\right)^{-1} \mathbf{H}^{*} \mathbf{v}_{n}=\mathbf{s}_{n}+\mathbf{R}^{-1} \mathbf{u}_{n} \\
& =\mathbf{R}^{-1}\left(\mathbf{R} \mathbf{s}_{n}+\mathbf{u}_{n}\right)=\mathbf{R}^{-1} \mathbf{y}_{n},
\end{aligned}
$$


where $\mathbf{u}_{n}=\mathbf{H}^{*} \mathbf{v}_{n}, \mathbf{y}_{n}=\mathbf{R} \mathbf{s}_{n}+\mathbf{u}_{n}=\mathbf{H}^{*} \mathbf{H} \mathbf{s}_{n}+\mathbf{H}^{*} \mathbf{v}_{n}=$ $\mathbf{H}^{*} \mathbf{r}_{n}$, and $\mathbf{R}=\mathbf{H}^{*} \mathbf{H}$, which is defined by

$\left[\begin{array}{ccccc}\left|h_{2}\right|^{2} & h_{2}^{*} h_{1} & h_{2}^{*} h_{0} & 0 & 0 \\ h_{1}^{*} h_{2} & \left|h_{1}\right|^{2}+\left|h_{2}\right|^{2} & h_{1}^{*} h_{0}+h_{2}^{*} h_{1} & h_{2}^{*} h_{0} & 0 \\ h_{0}^{*} h_{2} & h_{0}^{*} h_{1}+h_{1}^{*} h_{2} & \left|h_{0}\right|^{2}+\left|h_{1}\right|^{2}+\left|h_{2}\right|^{2} & h_{1}^{*} h_{0}+h_{2}^{*} h_{1} & h_{2}^{*} h_{0} \\ 0 & h_{0}^{*} h_{2} & h_{0}^{*} h_{1}+h_{1}^{*} h_{2} & \left|h_{0}\right|^{2}+\left|h_{1}\right|^{2} & h_{1}^{*} h_{0} \\ 0 & 0 & h_{0}^{*} h_{2} & h_{0}^{*} h_{1} & \left|h_{0}\right|^{2}\end{array}\right]$.

Apparently, $\mathbf{R}$ is a Hermitian matrix satisfying the condition $\mathbf{R}=\mathbf{R}^{*}$. Let us decomposite the matrix $\mathbf{R}$ into 2 matrices $\mathbf{R}=\mathbf{D}+\mathbf{R}_{\text {off }}$, where $\mathbf{D}$ is a diagonal matrix, and $\mathbf{R}_{\text {off }}$ is an off-diagonal matrix. For the matrix $\mathbf{R}$ expressed in (5), $\mathbf{D}$ and $\mathbf{R}_{\text {off }}$ are

$\mathbf{D}=\operatorname{diag}\left\{\left|h_{2}\right|^{2},\left|h_{1}\right|^{2}+\left|h_{2}\right|^{2},\left|h_{0}\right|^{2}+\left|h_{1}\right|^{2}+\left|h_{2}\right|^{2},\left|h_{0}\right|^{2}+\left|h_{1}\right|^{2},\left|h_{0}\right|^{2}\right\} ;$ $\mathbf{R}_{\text {off }}=\left[\begin{array}{ccccc}0 & h_{2}^{*} h_{1} & h_{2}^{*} h_{0} & 0 & 0 \\ h_{1}^{*} h_{2} & 0 & h_{1}^{*} h_{0}+h_{2}^{*} h_{1} & h_{2}^{*} h_{0} & 0 \\ h_{0}^{*} h_{2} & h_{0}^{*} h_{1}+h_{1}^{*} h_{2} & 0 & h_{1}^{*} h_{0}+h_{2}^{*} h_{1} & h_{2}^{*} h_{0} \\ 0 & h_{0}^{*} h_{2} & h_{0}^{*} h_{1}+h_{1}^{*} h_{2} & 0 & h_{1}^{*} h_{0} \\ 0 & 0 & h_{0}^{*} h_{2} & h_{0}^{*} h_{1} & 0\end{array}\right]$.

The matrix inversion in (4) can be solved iteratively by the Jacobi algorithm [10]

$$
\mathbf{s}_{n}^{i}=\mathbf{D}^{-1}\left(\mathbf{y}_{n}-\mathbf{R}_{\text {off }} \mathbf{s}_{n}^{i-1}\right)
$$

where $i$ is the iteration index. Substituting (6) into (7) yields $\mathbf{D}^{-1}=\operatorname{diag}\left\{1 /\left|h_{2}\right|^{2}, 1 /\left(\left|h_{1}\right|^{2}+\left|h_{2}\right|^{2}\right), \ldots 1 /\left|h_{0}\right|^{2}\right\}$

$\left[\begin{array}{c}s_{n-2}^{i} \\ s_{n-1}^{i} \\ s_{n}^{i} \\ s_{n+1}^{i} \\ s_{n+2}^{i}\end{array}\right]=\mathbf{D}^{-1}\left[\begin{array}{c}h_{2}^{*} r_{n} \\ h_{1}^{*} r_{n}+h_{2}^{*} r_{n+1} \\ h_{0}^{*} r_{n}+h_{1}^{*} r_{n+1}+h_{2}^{*} r_{n+2} \\ h_{0}^{*} r_{n+1}+h_{1}^{*} r_{n+2} \\ h_{0}^{*} r_{n+2}\end{array}\right]-\mathbf{D}^{-1}$
$\cdot\left[\begin{array}{c}h_{2}^{*} h_{1} s_{n-1}^{i-1}+h_{2}^{*} h_{0} s_{n}^{i-1} \\ h_{0}^{*} h_{2} s_{n-2}^{i-1}+\left(h_{0}^{*} h_{1}+h_{1}^{*} h_{2}\right) s_{n-1}^{i-1}+\left(h_{1}^{*} h_{0}+h_{2}^{*} h_{1}\right) s_{n+1}^{i-1}+h_{2}^{*} h_{0} s_{n+2}^{i-1} \\ h_{0}^{*} h_{2} s_{n-1}^{i-1}+\left(h_{0}^{*} h_{1}+h_{1}^{*} h_{2}\right) s_{n}^{i-1}+h_{1}^{*} h_{0} s_{n+2}^{i-1} \\ h_{0}^{*} h_{2} s_{n}^{i-1}+h_{0}^{*} h_{1} s_{n+1}^{i-1}\end{array}\right]$

The above procedure produces estimates of all the symbols in the vector $\mathbf{s}_{n}^{i}$. However, since detection of the central symbol $s_{n}$ relies on all the received samples in the vector $\mathbf{y}_{n}\left(\mathbf{r}_{n}\right)$, it can be assumed more accurate than the detection of the other symbols. From the above equation, we derive

$$
\begin{aligned}
s_{n}^{i}= & {\left[h_{0}^{*} r_{n}+h_{1}^{*} r_{n+1}+h_{2}^{*} r_{n+2}-h_{0}^{*} h_{2} s_{n-2}^{i-1}-\left(h_{0}^{*} h_{1}+h_{1}^{*} h_{2}\right) s_{n-1}^{i-1}\right.} \\
& \left.-\left(h_{1}^{*} h_{0}+h_{2}^{*} h_{1}\right) s_{n+1}^{i-1}-h_{2}^{*} h_{0} s_{n+2}^{i-1}\right] / \mathcal{P} \\
= & {\left[h_{0}^{*}\left(r_{n}-h_{2} s_{n-2}^{i-1}-h_{1} s_{n-1}^{i-1}\right)+h_{1}^{*}\left(r_{n+1}-h_{2} s_{n-1}^{i-1}-h_{0} s_{n+1}^{i-1}\right)\right.} \\
& \left.+h_{2}^{*}\left(r_{n+2}-h_{1} s_{n+1}^{i-1}-h_{0} s_{n+2}^{i-1}\right)\right] / \mathcal{P} \\
= & s_{n}+\underbrace{\left[h_{0}^{*} h_{2}\left(s_{n-2}-s_{n-2}^{i-1}\right)+\left(h_{0}^{*} h_{1}+h_{1}^{*} h_{2}\right)\left(s_{n-1}-s_{n-1}^{i-1}\right)\right] / \mathcal{P}}_{\text {cancellation residual }} \\
& +\underbrace{\left[\left(h_{1}^{*} h_{0}+h_{2}^{*} h_{1}\right)\left(s_{n+1}-s_{n+1}^{i-1}\right)+h_{2}^{*} h_{0}\left(s_{n+2}-s_{n+2}^{i-1}\right)\right] / \mathcal{P}}_{\text {cancellation residual }} \\
& +\underbrace{\left[h_{0}^{*} v_{n}+h_{1}^{*} v_{n+1}+h_{2}^{*} v_{n+2}\right] / \mathcal{P}}_{\text {noise }},
\end{aligned}
$$

where $\mathcal{P}=\sum_{i=0}^{2}\left|h_{i}\right|^{2}$. One can see from (8) that the decision statistic for the symbol $s_{n}$ at the $i^{t h}$ iteration is obtained by canceling the interference using the symbol estimates at the $(i-1)^{t h}$ iteration. With the decision statistic $s_{n}^{i}$, the symbol estimate can be obtained using the maximum likelihood decision rule $\hat{s}_{n}=\arg \min _{s_{m}}\left|s_{n}^{i}-s_{m}\right|^{2}$. In the case of QPSK modulation, $s_{m} \in\left\{s_{0}, s_{1}, s_{2}, s_{3}\right\}$. Note that at the beginning of the iterative process, no symbol estimates are available. We can use coherent non-cancellation detection to obtain an initial estimate of the transmitted symbols so that the interference cancellation can be carried out in the subsequent stage. This algorithm can be readily extended to a generic L-path channel.

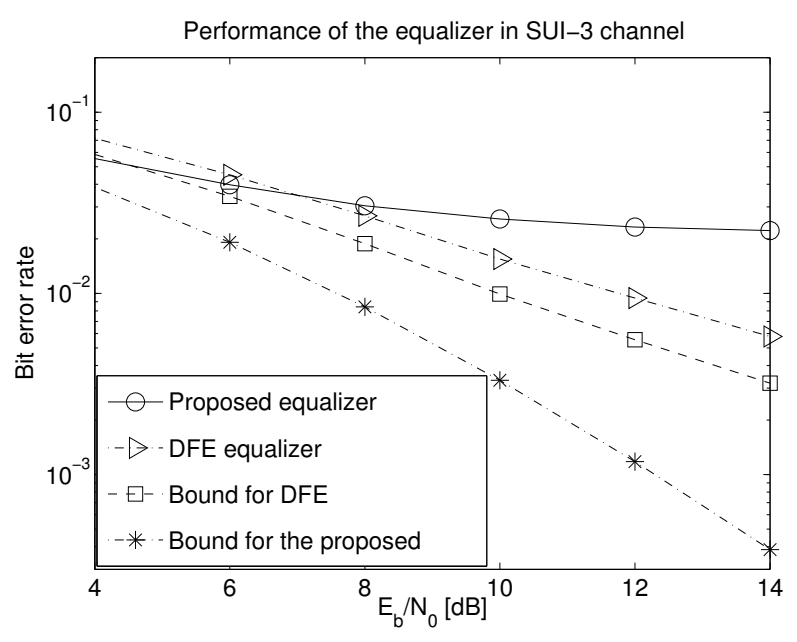

Fig. 1. Performance of the proposed equalizer and comparison with DFE and theoretical lower bounds. The curve for the proposed equalizer is plotted at the 3rd stage.

\section{B. Numerical results for the proposed equalizer}

Computer simulations are carried out to demonstrate the performance of the proposed algorithm. During each MonteCarlo run, the block size is set to 10000 bits, which correspond to 5000 QPSK symbols, 200 of which are used as pilot symbols for channel estimation. This is conducted using the modified maximum likelihood algorithm presented in [11]. We 7 choose the SUI-3 FWA channel introduced in [8]. The channel coefficients vary from one data block to another, however, they are assumed to remain constant during the transmission of one block of data. The simulated results are averaged over 1000 channel realizations.

In Fig. 1, we compare the performance of the proposed scheme with the conventional DFE equalizer with 5 feedforward, 3 feedback taps. It uses 200 pilots for training the equalizer coefficients (using recursive least square (RLS) adaptation). At high SNR, the conventional DFE performs better than the proposed equalizer. However, the proposed scheme has a much lower performance bound (represented by the dash-dot curve marked with asterisk computed by (12)) than that of the conventional DFE, thus exhibits a much better potential.

The performance of the proposed equalization scheme at different stages is illustrated in Fig. 2. The improvement by applying the Jacobi iterative algorithm over coherent noncancellation detection is significant if we compare the topmost solid curve with the other solid curves. It takes only 3 stages for the iterative scheme to converge. In Fig. 2, we also present the simulation results of the equalizer assuming i) perfect channel state information (CSI) and ii) the maximum likelihood channel estimation (CE), the details of which are presented in [11], and compare the simulation results with the results from theoretical analysis. In the simulations, the ISI is assumed to be known, and thus the cancellation is perfect. The theoretical curve is obtained by evaluating Eq. (12) numerically. The parameters settings are $s^{2}=0.36, \sigma^{2}=$ $0.175, \gamma_{1}=\mathrm{E}\left[\left|h_{1}\right|^{2}\right]=0.223, \gamma_{2}=\mathrm{E}\left[\left|h_{2}\right|^{2}\right]=0.07$ in (12). Fig. 2 shows that the theoretical analyses are in close agreement with the simulation results. Comparison between the simulation results with perfect CSI and the simulation results with $\mathrm{CE}$ indicate that the performance loss due to imperfect 


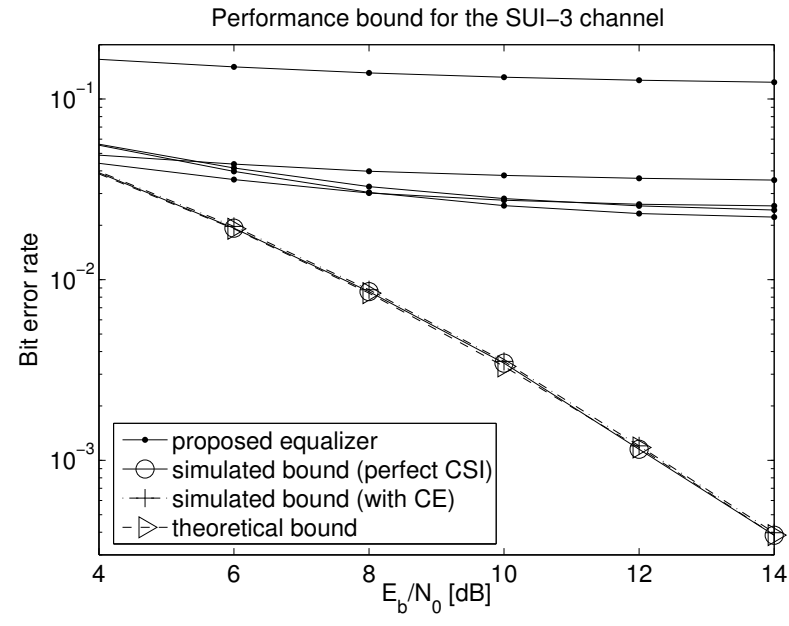

Fig. 2. Performance bound of the proposed equalizer: simulations vs. analyses, perfect CSI vs. CE. For proposed equalization, the topmost curve represents non-cancellation coherent detection stage and the bottommost curve represents the 4th equalization stage.

channel estimation is very small compared to the genie-aided case with perfect CSI.

One can see that the performance of the proposed scheme in uncoded systems is far above its performance bound. The rationale is that errors in the decision feedback significantly degrade performance and prevent the algorithm from reaching its theoretical potential. There are various ways of tackling this problem, e.g., using channel coding to reduce the feedback error probability, and/or using soft cancellation rather than brutal force cancellation to prevent error propagation. As will become evident in Section II-C, the theoretical potential of the proposed scheme can be realized by extending the algorithm to turbo equalization.

\section{A new approach to turbo equalization}

In order to reduce the error propagation and exploit the potential offered by the previously described equalization algorithm, we apply channel coding to the system, the baseband representation of which is depicted in Figure 3. The information sequence $\left\{b_{n}\right\}$ is convolutionally encoded into code bits $\left\{u_{n}\right\}$, which are subsequently interleaved and each block of two coded and interleaved bits $u_{n}^{\prime}[0], u_{n}^{\prime}[1]$ is mapped into one of the four QPSK symbols. The interleaver and deinterleaver are denoted as $\Pi$ and $\Pi^{-1}$, respectively, in Fig. 3 and Fig. 4. The QPSK symbol at time instant $n$ is denoted as $s_{n}=x_{n}+j y_{n}$. The QPSK symbols are transmitted over the ISI channel, which can be modeled as a $L$-tap transversal filter with impulse response $h_{n}=\sum_{l=0}^{L-1} h_{l} \delta_{n-l}$ where $h_{l}$ denotes the complex channel coefficients. The received signal is basically the same as (1) except that the symbols $\left\{s_{n-l}\right\}$ are now formed by coded bits rather than information bits.

The proposed turbo equalization algorithm is illustrated in Fig. 4. First, we use a training sequence to acquire a channel estimate $\hat{\mathbf{h}}$. In the meantime, a simple linear equalizer (LE) can be used to obtain an initial estimate of the transmitted symbols $\left\{\hat{s}_{n}=\hat{x}_{n}+j \hat{y}_{n}\right\}$. The channel estimate $\hat{\mathbf{h}}$ and symbol estimates $\left\{\hat{s}_{n}\right\}$ are passed to the equalizer (the SISO inner block shown in Fig. 4), which computes the log-likelihood ratio (LLR) value of $s_{n}$, denoted by $\lambda\left(s_{n}\right)=\lambda\left(x_{n}\right)+j \lambda\left(y_{n}\right)$. We use the equalization algorithm described previously, and apply the same method as described in [12] to derive LLR values $\lambda\left(x_{n}\right), \lambda\left(y_{n}\right)$ from the equalizer output. The LLR values of the symbols are mapped into LLR values of coded bits $\left\{\lambda\left(u_{n}^{\prime} ; O\right)\right\}$, which are deinterleaved to yield $\left\{\lambda\left(u_{n} ; I\right)\right\}$. For the QPSK modulated signals, the symbol LLR $\lambda\left(s_{n}\right)=$ $\lambda\left(x_{n}\right)+j \lambda\left(y_{n}\right)$ to bits LLRs $\lambda\left(u_{n}^{\prime}[0]\right), \lambda\left(u_{n}^{\prime}[1]\right)$ mapping rule is simply $\lambda\left(u_{n}^{\prime}[0] ; O\right)=\lambda\left(x_{n}\right), \lambda\left(u_{n}^{\prime}[1] ; O\right)=\lambda\left(y_{n}\right)$. Based on the soft input $\lambda\left(u_{n} ; I\right)$, a SISO outer channel decoder computes the LLR of each information bit $\lambda\left(b_{n} ; O\right)$ and each coded bit $\lambda\left(u_{n} ; O\right)$, where the former is used to make decisions on the transmitted information bit at the final iteration, and the latter is interleaved and passed through a bit-to-symbol converter (BSC) to derive a soft symbol estimate $\bar{s}_{n}=\bar{x}_{n}+j \bar{y}_{n}$, which is used for equalization at the next iteration. We use the notations $\lambda(\cdot ; I)$ and $\lambda(\cdot ; O)$ to denote the input and output ports of a SISO device. Several SISO algorithms can be used to compute the channel decoder outputs. For the purpose of this study, we consider the use of the Log-MAP algorithm [13].

The proposed scheme is evaluated and compared numerically with some existing algorithms in this section. In particular, we make a comparison with the MMSE filter based turbo equalization proposed by Tüchler, et. al. in [1] ${ }^{*}$, and the adaptive turbo equalization introduced by Laot, et. al. in [2]. The original algorithms were mostly derived for the BPSK modulated system or M-QAM system, have to be modified for the QPSK constellation under question. In the simulations, we employ a rate $1 / 3$ Maximum Free Distance convolutional code [5] with constraint length 5 and generator polynomials $(25,33,37)$ in octal form. During each Monte-Carlo run, the block size is set to 5000 information bits followed by 4 tails bits to terminate the trellis, which corresponds to $5004 \times 3=$ 15012 coded bits. They are interleaved by an $108 \times 139$ block interleaver and transmitted over a ISI channel. For the initial equalization stage of all the three schemes, we use a 7-tap linear MMSE equalizer, and 200 pilot symbols are used for training the equalizer. In the meantime, the modified maximum likelihood algorithm presented in [11] is used for channel estimation during the training period. Channel estimates are needed by the Laot's adaptive equalizer for the calculation of LLR values; they are also used for deriving filter coefficients and for interference cancellation by the other schemes. The simulation curves are obtained by averaging the simulation results over a minimum of 200 blocks of data transmitted and after at least 100 bit errors have occurred.

The performance comparison between the proposed scheme and some existing schemes is given in Fig. 5 and Fig. 6 for the SUI-3 channel and a 5-tap static channel, respectively. The impulse response of the static channel is defined as $h[n]=$ $(2-0.4 j) \delta[n]+(1.5+1.8 j) \delta[n-1]+\delta[n-2]+(1.2-$ $1.3 j) \delta[n-3]+(0.8+1.6 j) \delta[n-4]$, and the output channel power is normalized so that $P=\sum_{n=0}^{4}|h[n]|^{2}=1$. To study the behavior of each algorithm, the number of stages is set to 4 since it is observed that no more than 4 stages are needed for the discussed schemes to converge. For Laot's adaptive algorithm [2], the step size $\mu$ is set to 0.006 during the training period (200 pilots) and 0.002 during the tracking period. No exhaustive search has been performed to optimize the value of $\mu$, however, the chosen values have been found to perform

\footnotetext{
* The simulation results shown in this section are obtained by its original implementation. We have observed from our experiments that its approximate implementation with perfect a priori information has very close performance to the proposed scheme.
} 


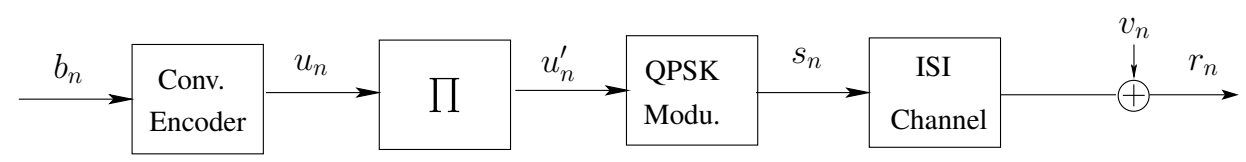

Fig. 3. Block diagram of a convolutionally coded QPSK system.

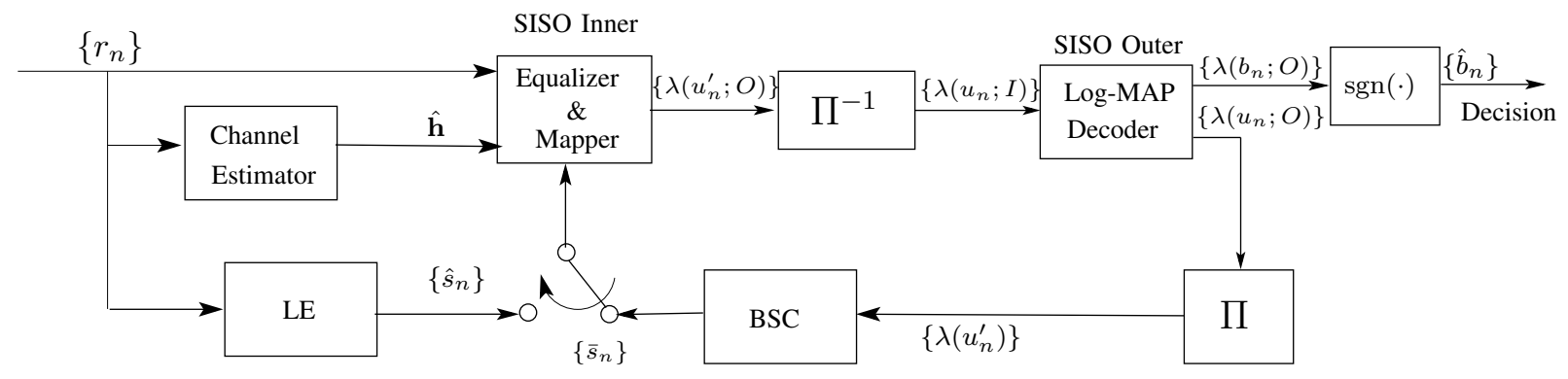

Fig. 4. Block diagram of the proposed turbo equalization scheme.

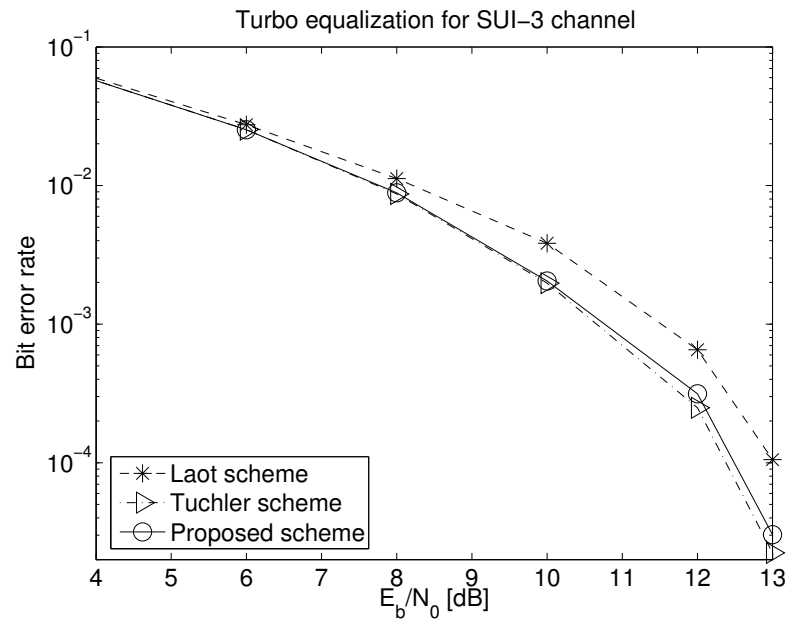

Fig. 5. Comparison of different turbo equalization algorithms after reaching convergence. All the curves represent the 4th stage turbo equalization.

reasonably well in various channel conditions. Results show that for the SUI-3/static channel, the proposed algorithm yields almost the identical/comparable results to the Tüchler's MMSE scheme, while achieves a gain of up to $0.5 \mathrm{~dB}$ compared to Laot's adaptive scheme after the system reaches convergence. The potential for performance improvement offered by the proposed scheme as predicted in Fig. 1 and Fig. 2 is realized by extending the algorithm to coded systems and by applying the turbo processing principle.

We also observed from our experiments that for channels with severe frequency-selectivity (e.g., Proakis D channel with impulse response $h[n]=0.1275 \delta[n]+0.450 \delta[n-1]+$ $0.750 \delta[n-2]+0.450 \delta[n-3]+0.1275 \delta[n-4])$, Tüchler's MMSE scheme has noticeable performance gain compared to the proposed scheme. The rationale is that when the condition of perfect cancellation is satisfied or approached, the proposed scheme does not incur a performance penalty compared to the MMSE scheme. However, when the bit error rate of the system is not very low, the condition of perfect cancellation cannot be approached, the performance of the proposed turbo equalization becomes suboptimum. It might be advantageous to apply Tüchler's MMSE scheme under such circumstances.

Table I shows the number of complex multiplications, divi-

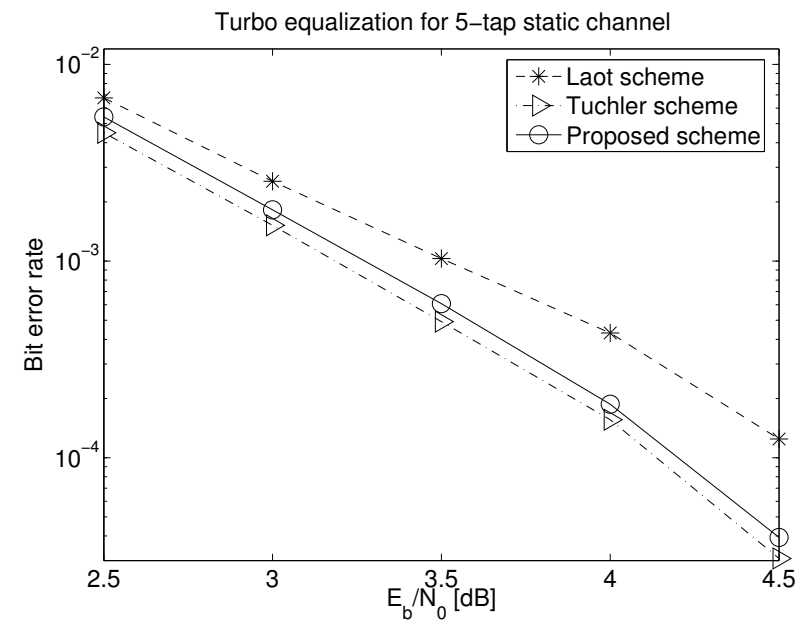

Fig. 6. Comparison of different turbo equalization algorithms for the static channel. All the curves represent the 4th stage turbo equalization.

TABLE I

COMPARISON OF COMPLEXITY FOR ONE SYMBOL ESTIMATE AT ONE ITERATION FOR THE ALGORITHMS CONSIDERED.

\begin{tabular}{|c|c|c|c|}
\hline \hline operations & multiplication & division & addition/subtraction \\
\hline Proposed & $L^{2}+6$ & 2 & $L^{2}+1$ \\
\hline Tüchler & $8 L^{3}-4 L^{2}+3 L+8$ & $2 L^{2}+2$ & $8 L^{3}-3 L^{2}+4 L$ \\
\hline Tüchler app. & $2 L^{2}+2 L+6$ & 2 & $2 L^{2}+2 L+1$ \\
\hline Laot & $6 L+4$ & 4 & $6 L+4$ \\
\hline
\end{tabular}

sions, and additions/subtractions required for the estimation of each QPSK symbol for the turbo equalization schemes considered, where $L$ is the number of channel taps. The figures for Tüchler and Laot's schemes are based on their modified versions which suit the QPSK modulation considered in this paper. For the Tüchler's scheme, both the original and its approximate implementation [1] are considered. One can see from the table that Laot's scheme has the lowest complexity, which is linear with $L$. However, it has the worst performance as one can see from the numerical results presented earlier. The proposed scheme reduces the complexity from $O\left(L^{3}\right)$ to $O\left(L^{2}\right)$ compared to the original Tüchler's scheme. It also has lower complexity than the approximate implementation of Tüchler's scheme. 


\section{CONClusions}

In this paper, we first introduced a new approach to equalization based on the Jacobi iterative algorithm. However, the results show that the performance of this algorithm is far from its theoretical potential in an uncoded system, due to the fact that errors in the decision feedback significantly degrade its performance. This suggests the use of channel coding to reduce the effect of feedback propagation errors. The idea of jointly equalizing and decoding of coded data over ISI channels leads to the proposed turbo equalization scheme, which is compared with the existing filter based equalization schemes, and is shown to achieve comparable performance with a reduced complexity. Numerical comparison indicates that the MMSE filtering is not necessary unless in severe ISI situation. The good performance and simplicity of this scheme makes it a feasible alternative for practical implementations, especially for the channels with large delay spreads.

\section{APPENDIX}

Here, we analyze the performance bound that can be achieved by the proposed equalization scheme in order to gain an insight into its asymptotic performance. The derivation is based on the assumption of perfect channel estimation and perfect cancellation. In this case, all the cancellation residuals will vanish, the equalizer output expressed by (8) only contains the desired signal and the noise, i.e., $z_{n}=\sum_{l=0}^{L-1}\left(\left|h_{l}\right|^{2} s_{n}+\right.$ $\left.h_{l}^{*} v_{n-l}\right) / \mathcal{P}=s_{n}+w_{n}$, where $\mathcal{P}=\sum_{l=0}^{L-1}\left|h_{l}\right|^{2}$, and $w_{n} \sim$ $\mathcal{C N}\left(0, N_{0} / \mathcal{P}\right)$. The bit error probability for the QPSK system is computed as [14]

$$
P_{b}=Q\left(\frac{A \sqrt{\sum_{l=0}^{L-1}\left|h_{l}\right|^{2}}}{\sqrt{N_{0} / 2}}\right)=Q\left(\sqrt{\frac{2 E_{b} \mathcal{P}}{N_{0}}}\right),
$$

where $Q(x)=\int_{x}^{\infty} \frac{1}{\sqrt{2 \pi}} \exp \left(-t^{2} / 2\right) d t$ is the complementary Gaussian cumulative distribution function. The above formula can be used directly for calculating the performance bound for static channels. Next, we use the SUI-3 channel [8] as an example to demonstrate how the performance bound for nonstatic channels can be calculated. For the 3-tap SUI-3 channel, $\mathcal{P}=\left|h_{0}\right|^{2}+\left|h_{1}\right|^{2}+\left|h_{2}\right|^{2}$. Let us denote $x=\left|h_{0}\right|^{2}, y=$ $\left|h_{1}\right|^{2}+\left|h_{2}\right|^{2}$. The bit error probability is a function of random variables $x$ and $y$, i.e.,

$$
P_{b}(x, y)=Q\left(\sqrt{\frac{2 E_{b}(x+y)}{N_{0}}}\right) .
$$

Since $\left|h_{0}\right|$, the amplitude of the first tap is Ricean distributed due to the existence of line of sight propagation [8], the random variable $x$ is non-central chi-square distributed with 2 degrees of freedom and PDF

$$
p(x)=\frac{1}{2 \sigma^{2}} \exp \left(-\frac{x+s^{2}}{2 \sigma^{2}}\right) I_{0}\left(\frac{\sqrt{x} s}{\sigma^{2}}\right), \quad x \geq 0,
$$

where $I_{0}(x)$ is the $0^{\text {th }}$ order modified Bessel function of the first kind [5, p. 44]. The amplitudes of the other two taps $\left(\left|h_{1}\right|,\left|h_{2}\right|\right)$ are characterized by a Rayleigh distribution [8]. Therefore, each of the random variables $\left|h_{1}\right|^{2},\left|h_{2}\right|^{2}$ has a central chi-square distribution with 2 degrees of freedom and characteristic functions $\psi_{\left|h_{1}\right|^{2}}(j v)=\left(1-j v \gamma_{1}\right)^{-1} ; \quad \psi_{\left|h_{2}\right|^{2}}(j v)=$ $\left(1-j v \gamma_{2}\right)^{-1}$, where $\gamma_{1}=\mathrm{E}\left[\left|h_{1}\right|^{2}\right], \gamma_{2}=\mathrm{E}\left[\left|h_{2}\right|^{2}\right]$, and $\gamma_{1} \neq \gamma_{2}$. As a consequence of the statistical independence of $\left|h_{1}\right|^{2}$ and $\left|h_{2}\right|^{2}$, the characteristic function of $y$ is

$$
\begin{aligned}
\psi_{y}(j v) & =\left(1-j v \gamma_{1}\right)^{-1}\left(1-j v \gamma_{2}\right)^{-1} \\
& =\frac{\gamma_{1}}{\gamma_{1}-\gamma_{2}}\left(1-j v \gamma_{1}\right)^{-1}+\frac{\gamma_{2}}{\gamma_{2}-\gamma_{1}}\left(1-j v \gamma_{2}\right)^{-1} .
\end{aligned}
$$

Taking the inverse Fourier transform, we obtain the PDF of $y$ as

$$
p(y)=\frac{1}{\gamma_{1}-\gamma_{2}} \exp \left(-\frac{y}{\gamma_{1}}\right)+\frac{1}{\gamma_{2}-\gamma_{1}} \exp \left(-\frac{y}{\gamma_{2}}\right), \quad y \geq 0 .
$$

To obtain the error probability when $x, y$ are random, we must average $P_{b}(x, y)$ expressed in (10) over the distribution of $x, y$, i.e., the average BER is calculated as

$$
\begin{aligned}
\bar{P}_{b}= & \int_{0}^{\infty} \int_{0}^{\infty} P_{b}(x, y) p(x) p(y) d y d x \\
= & \frac{1}{2 \sigma^{2}} \int_{0}^{\infty} \int_{0}^{\infty} Q\left(\sqrt{\frac{2 E_{b}(x+y)}{N_{0}}}\right) \\
& \cdot \exp \left(-\frac{x+s^{2}}{2 \sigma^{2}}\right) I_{0}\left(\frac{\sqrt{x} s}{\sigma^{2}}\right) \\
& \cdot\left[\frac{1}{\gamma_{1}-\gamma_{2}} \exp \left(-\frac{y}{\gamma_{1}}\right)+\frac{1}{\gamma_{2}-\gamma_{1}} \exp \left(-\frac{y}{\gamma_{2}}\right)\right] d y d x .
\end{aligned}
$$

\section{ACKNOWLEDGEMENT}

The financial support of the UK Engineering and Physical Sciences Research Council (under grant number EP/D07827X/1) is gratefully acknowledged.

\section{REFERENCES}

[1] M. Tüchler, R. Koetter, A. Singer. "Turbo equalization: principles and new results". IEEE Transactions on Communications, vol. 50, pp. 754 767, May 2002.

[2] C. Laot, A. Glavieux, Joel Labat. "Turbo equalization: adaptive equalization and channel decoding jointly optimized". IEEE Journal on Selected Areas in Communications, vol. 19, no. 9, Sept. 2001.

[3] C. Douillard, M. Jezequel, C. Berrou. "Iterative correction of intersymbol interference: turbo-equalization”. European Transactions on Telecommunications, pp. 507-511, Sept. 1995.

[4] L. Rasmussen, I. Oppermann. "Ping-pong effects in linear parallel interference cancellation for CDMA". IEEE Transaction on Wireless Communications, vol. 2, pp. 357-363, March 2003.

[5] J. Proakis. Digital Communications, 4th edition, McGraw-Hill, 2000.

[6] F. Hsu. "Square root Kalman filtering for high-speed data received over fading dispersive HF channels". IEEE Transactions on Information Theory, vol. 28, pp. 753-763, Sept. 1982.

[7] IEEE 802.16 Working Group on Broadband Wireless Access Standards. available at http://grouperieee.org/groups/802/16/.

[8] V. Erceg et al. "Channel models for fixed wireless applications". IEEE 802.16a cont. IEEE 802.16.3c-01/29r4, June 2003.

[9] S. Kay. Fundamentals of statistical signal processing, Prentice Hall, NJ, 1998.

[10] O. Axelsson. Iterative Solution Methods, Cambridge University Press, 1994.

[11] P. Xiao, R. Carrasco, I. Wassell. "Estimation of FWA MIMO channels". Proceedings IEEE Information Theory Workshop, ITW'2006, pp. 641645, Oct. 2006.

[12] P. Xiao, R. Carrasco, I. Wassell. "Turbo equalization for space-time block coded FWA systems". Proceedings IEEE Information Theory Workshop, ITW'2006, pp. 626-630, Oct. 2006.

[13] P. Robertson, E. Villebrun, P. Hoeher. "A comparison of optimal and sub-optimal MAP decoding algorithms operating in the log domain". Proc. IEEE International Conference on Communications, pp. 10091013, 1995.

[14] P. Xiao, R. Carrasco, I. Wassell. "Performance analysis of conventional detection in BFWA systems". Proc. Second IFIP International Conference on Wireless and Optical Communications Networks, WOCN'2005, pp. 447-452, March 2005. 